\title{
High level MycN expression in non-MYCN amplified neuroblastoma is induced by the combination treatment nutlin-3 and doxorubicin and enhances chemosensitivity
}

\author{
SUSAN K. PEIRCE and HARRY W. FINDLEY

\begin{abstract}
Department of Pediatrics, Division of Hematology and Oncology, Emory University School of Medicine,
\end{abstract} \\ Aflac Cancer Center and Blood Service, 2015 Uppergate Drive, Atlanta, GA 30322, USA
}

Received March 31, 2009; Accepted June 26, 2009

DOI: $10.3892 /$ or_00000586

\begin{abstract}
M Y C N$ gene amplification is a negative prognostic indicator in neuroblastoma and high level $\mathrm{MycN}$ expression in Stage IV neuroblastoma is generally a hallmark of poor patient outcome. However, high level expression of the $\mathrm{MycN}$ protein in neuroblastoma cells lacking $M Y C N$ amplification suppresses growth and drives apoptosis; this, in part, explains the absence of clinical observations of high level $\mathrm{MycN}$ in neuroblastoma lacking $M Y C N$ amplification. In the current study, we found that combination treatment with nutlin-3 and doxorubicin upregulated $\mathrm{MycN}$ expression in non- $M Y C N$ amplified neuroblastoma cells at both the protein and mRNA levels. The induced expression of $\mathrm{MycN}$ in non- $M Y C N$ amplified cells inhibited cell proliferation and increased apoptosis. MycN induction also upregulated p53, p21 and Bax protein levels, as well as mRNA levels for the positive neuroblastoma prognostic factors CD44 and EFNB3. Blocking MycN reversed these effects. These results were corroborated by findings using a $\mathrm{MycN}$-inducible system in SHEP cells, another $M Y C N$ non-amplified neuroblastoma cell line. Our results indicate that doxorubicin/nutlin-3 combination treatment both induces expression of $\mathrm{MycN}$ in a non-MYCN-amplified background and sensitizes neuroblastoma cells to chemotherapy. These findings support the idea that induction of $\mathrm{MycN}$ in non- $M Y C N$-amplified cells drives neuroblastoma cells toward apoptosis and suggest that combination nutlin-3/doxorubicin treatment may be clinically important.
\end{abstract}

\section{Introduction}

Neuroblastoma (NB) is a childhood solid tumor of the peripheral nervous system arising from neural crest progenitor

Correspondence to: Dr Susan K. Peirce, Department of Pediatrics, Division of Hematology and Oncology, Emory University School of Medicine, Aflac Cancer Center and Blood Service, 2015 Uppergate Drive, Atlanta, GA 30322, USA

E-mail: susan_peirce@oz.ped.emory.edu

Key words: neuroblastoma, nutlin-3, MycN cells and the most common pediatric solid tumor in children under five years of age; it is thought to arise from improper differentiation of neural crest cells. One of the many enigmas of NB is that most infants experience complete regression of the disease even without therapeutic intervention, while older children have a far more unfavorable outcome, with metastatic disease that is often unresponsive to intense therapy. In addition, initial responsiveness to chemotherapy may sometimes lead to relapse and drug resistance (1).

In addition to younger age, there are other favorable prognostic factors, including lack of $M Y C N$ amplification (2), early tumor stage and expression of the genes EPHB6, EFNB2 and EFNB3 (3); these are members of the ephrin family, receptor protein-tyrosine kinases involved in the development of the nervous system. Expression of the cell-surface glycoprotein CD44 has been found to be a statistically reliable independent predictor of event-free survival (2) and aggressive NB tumors with $M Y C N$ amplification have been shown to downregulate the expression of CD44 (4). High level expression of EPHB3, EFNB2, EFNB3 and CD44 correlate with low-stage NB (5) and forced expression of these genes inhibits the growth of NB cells (3). Additionally, expression of EPHB6, EFNB2 and EFNB3 genes correlates with high levels of the neurotropin receptor TrkA, another favorable prognostic factor (6). Thus, these molecular markers can be used in combination with other factors in a predictive model of favorable NB outcome.

In contrast, the deregulated high level expression of $\mathrm{MycN}$ in conjunction with $M Y C N$ amplification is prognostic of poor patient outcome in NB (reviewed in ref. 7). The expression of $\mathrm{MycN}$ is essential during normal neural crest development, but it is downregulated as tissues terminally differentiate (8). The critical E2F-1, 2, 3 and 4 transcription regulators bind in the autoregulatory site of the $\mathrm{MycN}$ promoter and have been shown to upregulate or repress $\mathrm{MycN}$ expression (9), in addition to factors that alter the chromatin states of the $\mathrm{MycN}$ promoter (10). The role $M Y C N$ amplification plays in NB tumorigenesis was confirmed in a pivotal early study using transgenic mice that overexpress $\mathrm{MycN}$ in neuroectodermal cells and develop neuroblastoma (9). In the context of NB malignancy, $\mathrm{MycN}$ is a transcription factor that heterodimerizes with Max to interact with E-box elements (10) within promoters of genes generally associated with proliferation and drug resistance, thus upregulating expression of proteins such 
as MRP1 (11) and insulin-like growth factor (12). In this manner, MycN has been shown to enhance NB cell growth and tumorigenicity. $M Y C N$ amplification also correlates with a failure to arrest in G1 (13). A number of studies have linked coexpression of high $\mathrm{MycN}$ levels with high level expression of other negative prognostic NB genes (14), including pAkt (15), VEGF (16), livin (17) and MDM2, the p53 regulatory gene which has been found to be a transcriptional target of $\mathrm{MycN}$ (18). Conversely, the knock-down of MycN expression in some $M Y C N$-amplified NB cells is associated with caspase-3 activation and apoptosis (19) as well as neuronal cell differentiation (20).

However, the prognostic significance of $\mathrm{MycN}$ overexpression in NB appears to depend on $M Y C N$-amplification status. A number of studies have shown that high level $\mathrm{MycN}$ expression in the absence of amplification is associated with a favorable outcome $(5,21,22,24)$. Indeed, conditional expression of $\mathrm{MycN}$ in some non- $M Y C N$-amplified NB cell lines has been shown to significantly inhibit cell growth and to induce apoptosis $(23,26)$. Conversely, in another nonamplified NB cell line, it was observed that neither forced expression of $\mathrm{MycN}$ nor the use of cytotoxic drugs induces apoptosis, but that the two interact synergistically (25) and that this response is dependent on mitochondrial permeability and the activation of caspases. Another study supporting the role of caspases in $\mathrm{MycN}$-induced apoptosis includes the observations that $M Y C N$ amplification is often independently associated with methylation of caspase- 8 and with defects in tumor factor-related apoptosis inducing ligand (TRAIL); ectopic MycN expression in non-MYCN-amplified cell lines induces both caspase- 8 and caspase-9-mediated apoptosis (26).

In this study, we initially investigated the ability of nutlin-3 to increase sensitivity to doxorubicin in a non-MYCN-amplified NB cell line, SH-SY5Y. We demonstrate marked induction of MycN in SH-SY5Y cells at both mRNA and protein levels following combination treatments. Significantly, MycN induction was accompanied by increased sensitivity to doxorubicin in the presence of nutlin-3. This interaction was corroborated using a $\mathrm{MycN}$-inducible cell system in conjunction with combination treatments. Our findings suggest a novel and distinct pathway involved in chemotherapy-induced NB apoptosis. Our results also extend previous findings showing that chemotherapy in combination with forced expression of $\mathrm{MycN}$ in non-amplified MYCN NB cells induces apoptosis $(25,27)$.

\section{Materials and methods}

Reagents and primers. Nutlin-3 (cat. no. 10004372) was purchased from Cayman Chemical (Ann Arbor, MI) and represents an equimolar mix of the active (nutlin-3a) and inactive (nutlin-3b) isomers. In this study, all molar concentrations are expressed in terms of nutlin-3a. Doxorubicin was obtained from the pharmacy of Egleston Hospital of Emory University. EFNB3-specific forward and reverse primer sequences are: 5'-ctgaaatgcccatggaaaga-3'; and 5'-acgcccagc aagagcagcgc- $3^{\prime}$. The forward and reverse primer sequences for CD44 are: 5'-ccgctatgtccagaaaggag-3'; 5'-tccagggactgtctt cgtct- 3 '. MycN forward and reverse sequences are: 5'-cggtcc cccacctctctt-3'; 5'-cggtttagccaccaactttctc-3'.
Cell lines, light microscopy and siRNA silencing. SH-SY5Y cell lines were maintained in RPMI-1640 medium $+10 \%$ FBS. Cells were photographed with an Olympus IX50. The SHEP Tet21N MYCN expression system previously described (28) was used to conditionally express $\mathrm{MycN}$ in a non-MycNamplified background. Cells were grown in RPMI-1640 containing hygromycin at $25 \mu \mathrm{g} / \mathrm{ml}$ and supplemented with $10 \%$ tetracycline-free FCS (Clontech, Mountainview, CA). To block expression of $\mathrm{MycN}, 10 \mathrm{ng} / \mathrm{ml}$ of tetracycline was added to growth media for at least $24 \mathrm{~h}$. siRNA (Stealth Prevalidated cat. no.46454497) for MycN knock-down and the Stealth Negative Control (cat. no. 12935) were purchased from Invitrogen Life Technologies (Carlsbad, CA) and the target sequence for $\mathrm{MycN}$ is: 5'-ccacgugccggaguugguaaa gaau-3'. Cells were plated $24 \mathrm{~h}$ before transfection at a concentration of $2 \times 10^{4}$ per mm well (96-well plate) or $3 \times 10^{5}$ per $35 \mathrm{~mm}^{2}$ well; siRNA or the negative control were transfected using Oligofectamine (Invitrogen; cat. no. 12252) at 5 pmole (96-well plate) or 200 pmole (35 mm well), per manufacturer's instructions. Cells were assayed at 24, 48 and $72 \mathrm{~h}$ post transfection.

Western blotting and antibodies. For Western blotting, cells were lysed in buffer containing $20 \mathrm{mM}$ Tris- $\mathrm{HCl}, 150 \mathrm{mM}$ $\mathrm{NaCl}, 1 \mathrm{mM}$ Na2EDTA, $1 \mathrm{mM}$ EGTA, 1\% Triton, $2.5 \mathrm{mM}$ sodium pyrophosphate, $1 \mathrm{mM}$ B-glyceraldehyde, $1 \mathrm{mM}$ $\mathrm{Na}_{3} \mathrm{VO}_{4}, 1 \mu \mathrm{g} / \mathrm{ml}$ leupeptin, $1 \mathrm{mM}$ PMSF. Thirty-fifty $\mu \mathrm{g} /$ well of cleared lysate were run on 10 or $12.5 \%$ Tris/glycine PAGE gels and transferred to NC under standard conditions. The following primary antibodies were purchased from Santa Cruz Biotechnology (Santa Cruz, CA): anti-p53 FL-393 (sc-6243), anti-Bax (sc-20067), anti-E2F1 (SC-193) and anti-p21 (sc-817). Phospho-MDM2 (\#3521) was purchased from Cell Signaling Technology (Beverly, MA). Anti-ß-actin (AC-74) was purchased from Sigma (St. Louis, MO). Anti-N-Myc antibody (OP-13) was purchased from EMD Calbiochem (Darmstadt, Germany). All primary antibodies were used at manufacturer's suggested concentrations. Goat anti-mouse or rabbit HRP-conjugated secondary antibodies (Santa Cruz Biotechnology) were used at 1:2000.

Real-time RT-PCR. A two-step real-time RT-PCR procedure was used. All reagents were purchased from Qiagen (Valencia, CA) and all methods used were those recommended by the manufacturer. Total RNA was isolated from cells using QIAshredders (cat. no. 79654) and RNeasy (cat. no. 74104). For RT, samples were incubated at $42^{\circ} \mathrm{C}$ using Qiagen Quanti Tec RT reagents. SYBR-Green reagents (cat. no. 204143) were used for the 40 cycle real-time PCR and reactions were run on an Applied Biosystems 7500 Real-Time PCR machine. GADPH was used to normalize levels of mRNA for the relative quantification method of analysis.

Cell proliferation and apoptosis. The tetrazolium-based colorimetric assay, WST-1 (cat. no. 11644807, Roche Applied Science, Mannheim, Germany), was used for the quantification of cell proliferation. Cells were plated at $2 \times 10^{4}$ cells/well of a 96-well plate and treatments were performed in triplicate. Measurements were made at $450 \mathrm{~nm}$ with a reference wavelength of $600 \mathrm{~nm}$. The Cell Death Detection ELISA ${ }^{\text {PLus }}$ kit 
(cat. no. 11774425, Roche Applied Science) was used to quantitate levels of mono- and oligonucleosomes in the cytoplasm of apoptotic cells after treatments, following the manufacturer's instructions, with the following modification: $10^{3}$ cells were used per well of a 96-well plate. Measurements were made at $405 \mathrm{~nm}$ with a reference wavelength of $490 \mathrm{~nm}$.

Statistical analyses. All experiments were repeated independently at least three times. Where shown, results are presented as mean \pm SE. Statistical analyses were conducted using Student's t-test. Group differences resulting in P-values of $<0.05$ were considered to be statistically significant. ${ }^{*} \mathrm{P}<0.05$, ${ }^{* * *} \mathrm{P}<0.01,{ }^{* * *} \mathrm{P}<0.005$.

\section{Results}

Nutlin-3 and doxorubicin interact to inhibit cell proliferation, induce apoptosis and substantially upregulate the expression of MycN in SH-SY5Y cells. To gain an initial understanding of the interactive effects of the combination treatment on this non- $M Y C N$-amplified cell line, we evaluated these responses using proliferation and apoptosis assays and surveyed changes in protein expression. Using WST-1 assays, we found that the combination treatment significantly reduced proliferation over lower levels of either single treatment (Fig. 1A, top). The $\mathrm{IC}_{50}$ of $0.035 \mu \mathrm{M}$ for doxorubicin alone decreased to an $\mathrm{IC}_{50}$ of $0.014 \mu \mathrm{M}$ at both concentrations of nutlin-3.

The ELISA apoptosis assay quantifies levels of histonecomplexed DNA fragments. Using this assay, we found that while higher doses of the doxorubicin single treatment or the combination treatments both produced increasing levels of apoptosis, the highest dose of the combination treatments induced apoptosis at levels almost 2-fold higher than the highest single dose of doxorubicin (Fig. 1A, bottom). Both single and combination treatments induced the upregulation of p53, Bax and pMdm2. However, only the combination treatment drove the high level expression of the $\mathrm{MycN}$ protein (Fig. 1B, top). Moreover, the combination treatment upregulated the expression of the transcriptional factor E2F1 (Fig. 1B, bottom).

Treatment with nutlin-3 and doxorubicin promotes morphological changes in SH-SY5Y cells consistent with apoptosis. SH-SY5Y is a tumorigenic N-type NB cell line, resembling neuroendocrine precursors. We examined changes in cell morphology following a $24 \mathrm{~h}$ treatment with single and combination treatments. The doxorubicin treatment alone promoted an increase in cell size (Fig. 2, panel B), while the nutlin-3 treatment induced neuritic-like outgrowths resembling differentiation (Fig. 2, Panel C). The combination treatment induced more pronounced outgrowths, as well as a strongly refractile appearance characteristic of apoptosis (Fig. 2, Panel D).

The combination treatment markedly induces MycN mRNA, upregulates pro-apoptotic proteins and enhances the expression of favorable neuroblastoma genes, CD44 and $E F N B 3$. To gain insight into the time-dependent nature of gene and protein regulation following the combination treatment, we examined MycN, CD44 and EFNB3 mRNA expression
A
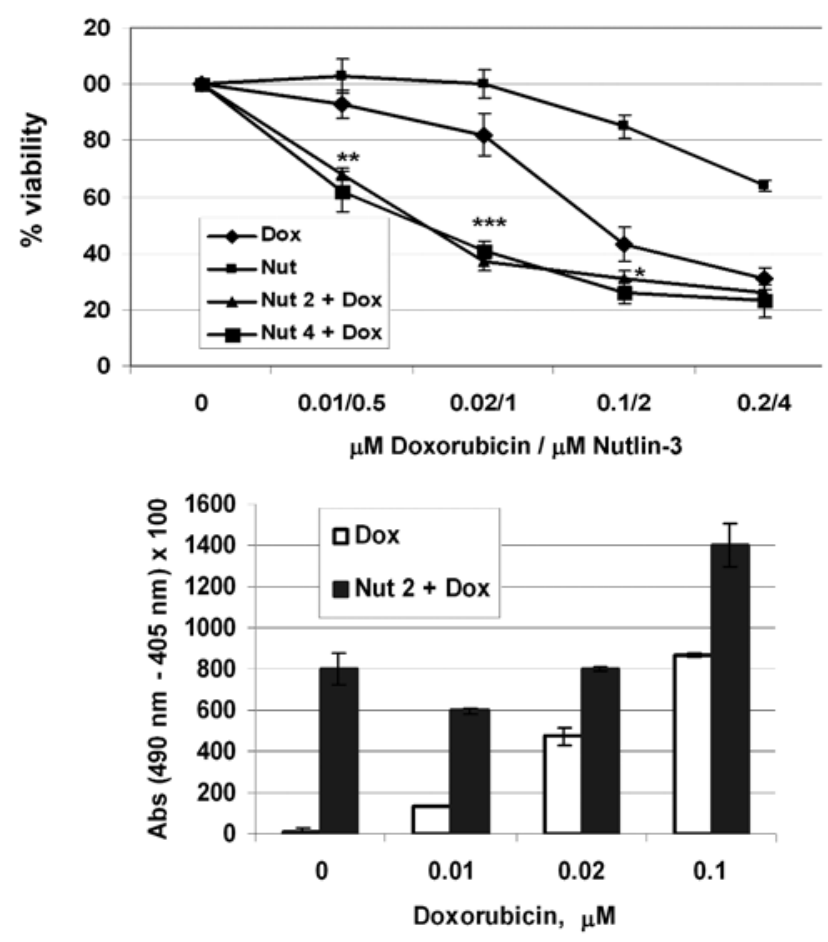

B

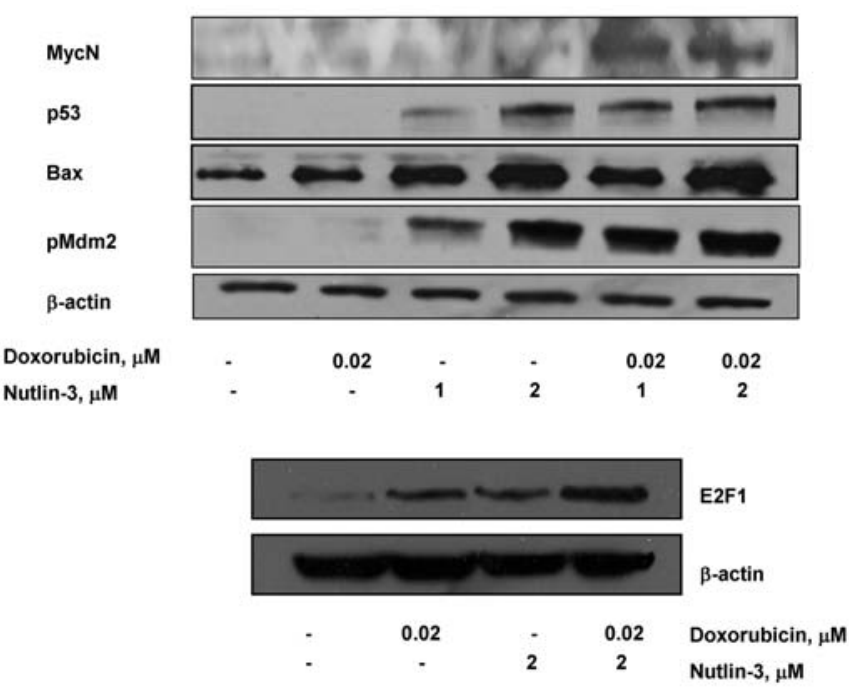

Figure 1. Nutlin-3 and doxorubicin interact to inhibit cell proliferation, induce apoptosis and upregulate the expression of MycN in SH-SY5Y cells. (A) Top panel, dose response for single and combination treatments, as measured by $24 \mathrm{~h}$ WST-1 assay, indicating a significant decrease in viability at lower doses of combination treatments vs. single treatments. Bottom panel, ELISA apoptosis assay using quantification of mono- and oligonucleosomes in cytoplasm as measure of apoptosis. Results are representative and show one experiment performed in triplicate. (B) Top panel, $24 \mathrm{~h}$ combination treatments of doxorubicin and nutlin-3 induce expression of $\mathrm{MycN}$ protein in a non-MycN amplified cell line; single treatments do not. Bottom panel $24 \mathrm{~h}$ combination treatment of doxorubicin and nutlin-3 upregulates expression of E2F1.

and p53 and Bax protein expression. In response to the combination treatment, MycN mRNA was strongly upregulated at an early time point, but this expression decreased through $30 \mathrm{~h}$ (Fig. 3A, top panel). Simultaneously, p53 protein expression increased markedly by $4 \mathrm{~h}$ and remained elevated 


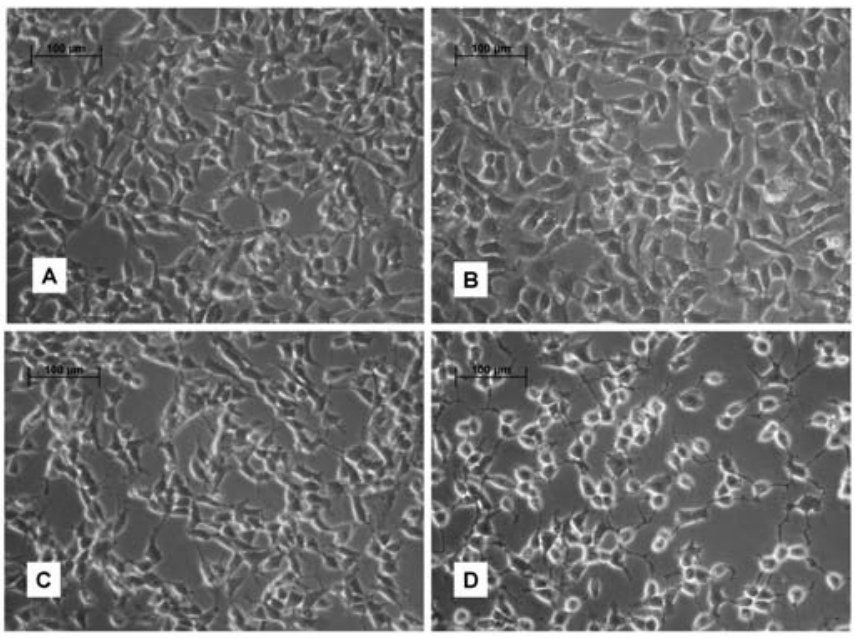

Figure 2. The 24 h doxorubicin/nutlin-3 combination treatment of SH-SY5Y cells promotes alterations in cell morphology consistent with apoptosis. (A) No treatment; (B) treatment with $0.02 \mu \mathrm{M}$ doxorubicin; (C) treatment with $4 \mu \mathrm{M}$ nutlin-3; and (D) treatment with $4 \mu \mathrm{M}$ nutlin-3 $+0.02 \mu \mathrm{M}$ doxorubicin.

through $30 \mathrm{~h}$ (Fig. 3A, bottom panel). In contrast, Bax protein levels rose gradually over this same time period and were highest at $30 \mathrm{~h}$ (Fig. 3A, bottom panel).

CD44 expression has been found to inversely correlate with $M Y C N$ amplification and thus functions as a positive prognostic factor for NB. EFNB3 is a ligand member of the ephrin receptor protein-kinase gene family and is important in neural development; its expression has also been found to correlate with a positive NB outcome. Therefore, we sought to examine the expression of these genes in response to induced $\mathrm{MycN}$ expression. CD44 gene expression was highest at $24 \mathrm{~h}$ ( $\sim 10$-fold higher than untreated cells) but decreased by $30 \mathrm{~h}$ (Fig. 3B, top panel). Conversely, EFNB3 levels were somewhat elevated by $8 \mathrm{~h}(\sim 1.4$-fold higher than untreated cells) and remained at this level through $24 \mathrm{~h}$ (Fig. 3B, bottom panel).

Blocking MycN mRNA prevents combination-treatmentinduced increases in expression of Bax protein and partially reverses apoptosis. To evaluate the role of increased $\mathrm{MycN}$ in the upregulation of Bax protein expression, we used siRNA against $M Y C N$ to inhibit $\mathrm{MycN}$ expression following combination treatment. At $8 \mathrm{~h}$ following treatment, $\mathrm{MycN}$ levels were substantially reduced from the $\mathrm{MycN}$ levels of the negative control RNA transfection (Fig. 4A, top panel); by $24 \mathrm{~h}$, levels of MycN mRNA were comparable for the negative control and $\mathrm{MycN}$-targeted transfections. From Fig. 4A, bottom panel and Fig. 3A (bottom panel), there was an increase in levels of Bax protein between 8-24 h following the combination treatment. Evidence that $\mathrm{MycN}$ is responsible for this upregulation was supported by the lack of increased Bax induction following inhibition of MycN mRNA expression (Fig. 4A, bottom).

To confirm that MycN plays a role in driving apoptosis in these combination treatments, we measured levels of cleaved nucleosomes following siRNA-induced $\mathrm{MycN}$ silencing, using an ELISA assay. For all three treatments, levels of apoptosis were reduced when $\mathrm{MycN}$ RNA expression was inhibited (Fig. 4B).

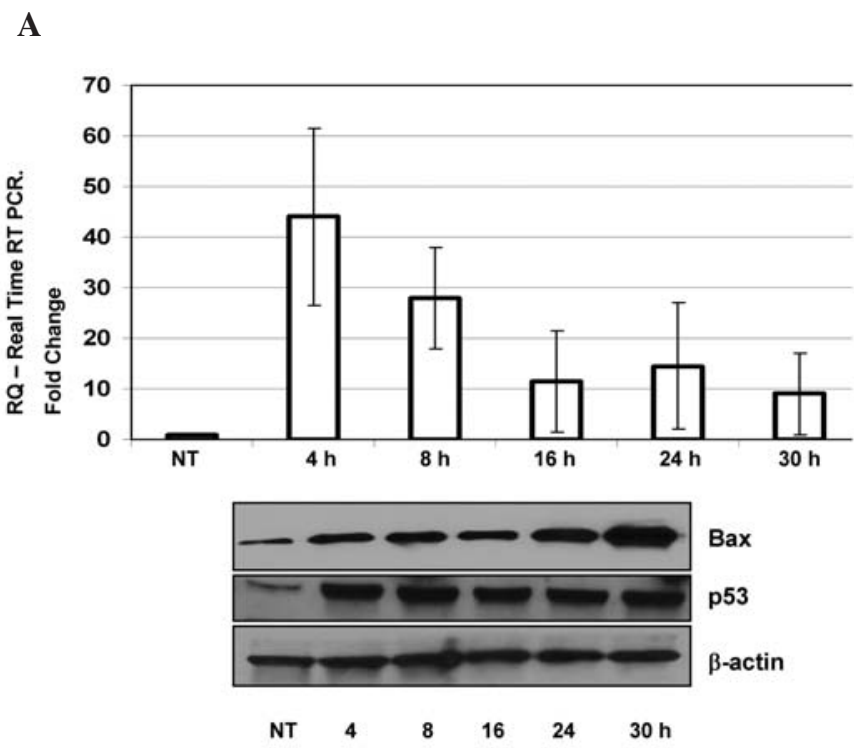

B
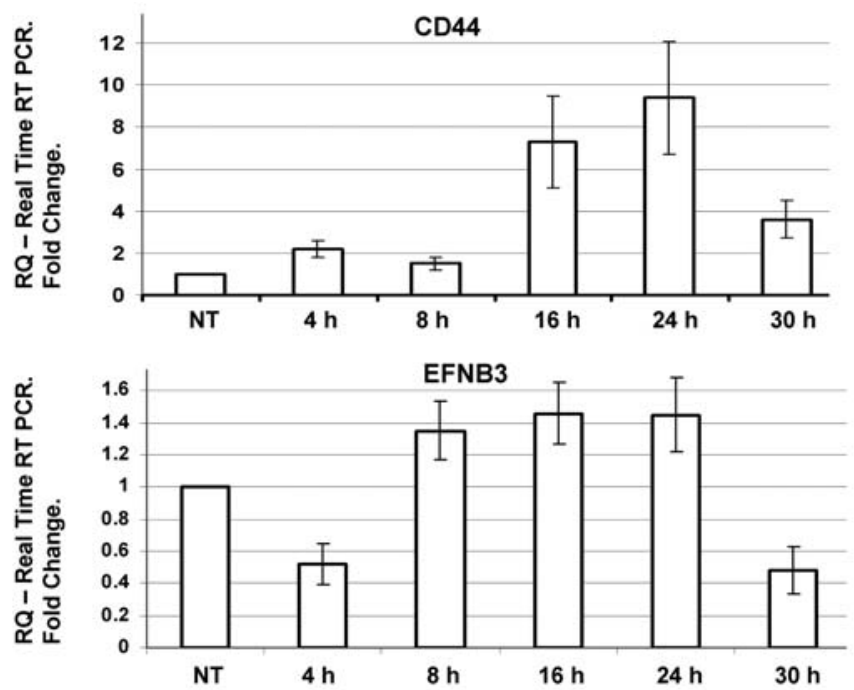

Figure 3. The combination treatment $2 \mu \mathrm{M}$ nutlin-3 $+0.02 \mu \mathrm{M}$ doxorubicin strongly induces $\mathrm{MycN}$ mRNA, upregulates downstream apoptotic proteins and enhances the mRNA expression of genes favorable to neuroblastoma outcome. (A) Top panel, MycN mRNA expression levels are significantly increased by $4 \mathrm{~h}$ following treatment, as measured by real-time RT-PCR. Results are representative and show one experiment run in triplicate. Bottom panel, p53 protein levels are fully upregulated by $4 \mathrm{~h}$, while Bax expression continues to increase through $30 \mathrm{~h}$. (B) Induction of $\mathrm{MycN}$ by combination treatment in a non-MycN amplified cell line corresponds to increased mRNA expression of two cell surface markers associated with a positive neuroblastoma prognosis, CD44 and EFNB3. Real-time RT-PCR results are representative and show one experiment performed in triplicate.

In a MycN-inducible cell line (SHEP-Tet21N), MycN interacts with combination treatments to increase levels of pro-apoptotic proteins and enhance the inhibition of proliferation. To evaluate the interactive effects of the combination treatments and the expression of the $\mathrm{MycN}$ protein, we used an inducible system of $\mathrm{MycN}$ in a non-MYCN-amplified cell line (SHEP). $\mathrm{MycN}$ protein expression was undetectable in the presence of $10 \mathrm{ng} / \mathrm{ml}$ of tetracycline but expressed at high levels in its absence (Fig. 5A, top lane). Levels of p53 were increased in 
A
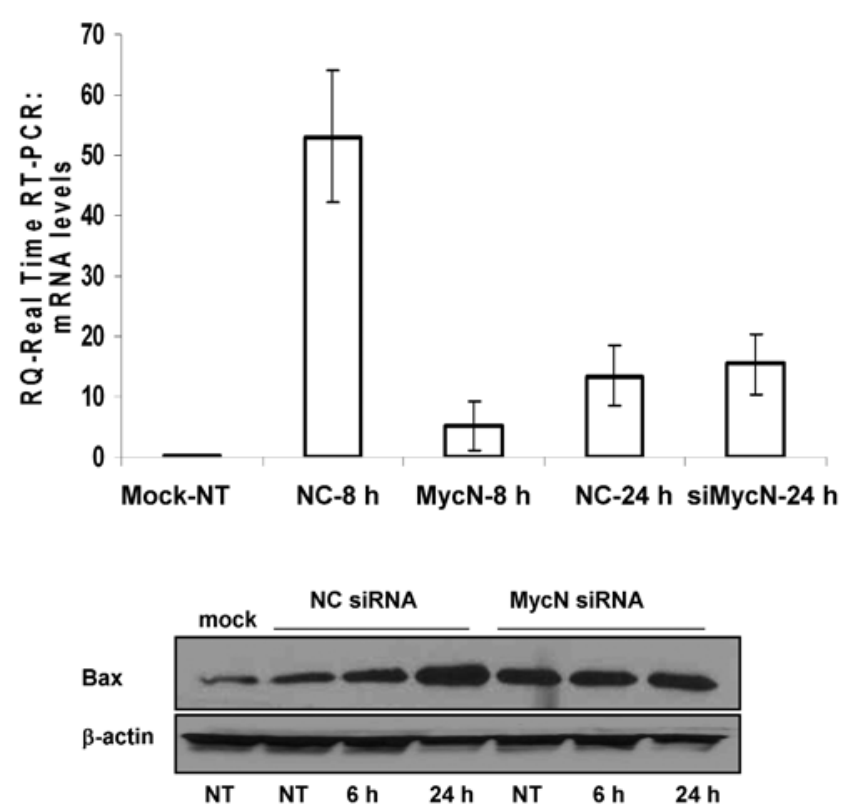

B

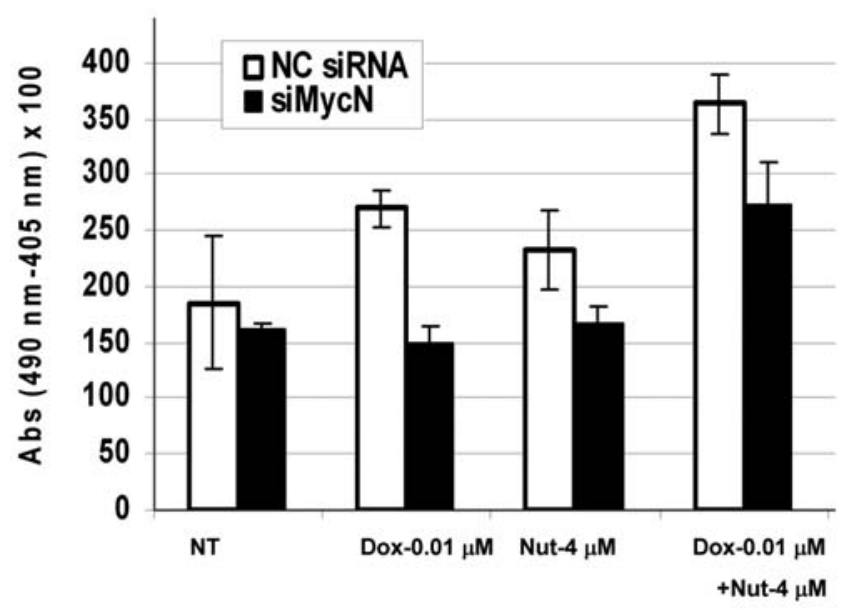

Figure 4. Transfection with MycN siRNA preceeding treatment with $2 \mu \mathrm{M}$ nutlin- $3+0.02 \mu \mathrm{M}$ doxorubicin blocks the increased expression of Bax protein and partially inhibits apoptosis. (A) Top panel, MycN mRNA levels are moderately suppressed by MycN siRNA transfection at $8 \mathrm{~h}$, but return to higher levels by $24 \mathrm{~h}$. A $24 \mathrm{~h}$ combination treatment followed an $18 \mathrm{~h}$ transfection. NC, negative control siMycN. Real-time RT-PCR results are representative and show one experiment performed in triplicate. Bottom panel, transfection with $\mathrm{MycN}$ siRNA in combination-treated cells leads to suppression of Bax upregulation. A $24 \mathrm{~h}$ combination treatment followed an $18 \mathrm{~h}$ transfection. (B) ELISA apoptosis assay using quantification of monoand oligonucleosomes in cytoplasm as measure of apoptosis. Apoptosis levels were measured following an $18 \mathrm{~h}$ transfection and an additional $24 \mathrm{~h}$ combination treatment. Results are representative and show one experiment performed in triplicate.

response to the combination treatment in the absence of $\mathrm{MycN}$. However, in the presence of $\mathrm{MycN}$, levels of p53, as well as its two downstream effector targets, p21 and Bax, were dramatically increased in response to combination treatments from levels seen without expression of $\mathrm{MycN}$ (Fig. 5A).

To examine a mechanistic role for the $\mathrm{MycN}$ protein, we used this cell system to evaluate viability in the presence or absence of $\mathrm{MycN}$ with single and combination treatments.
$\mathbf{A}$

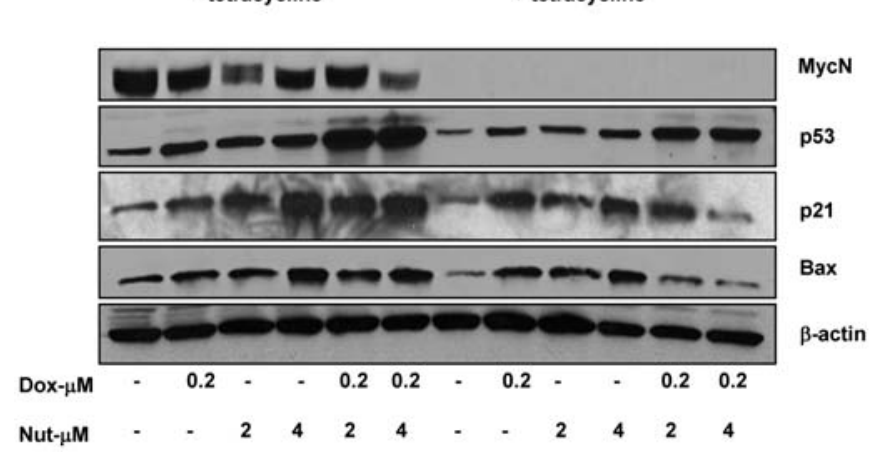

B

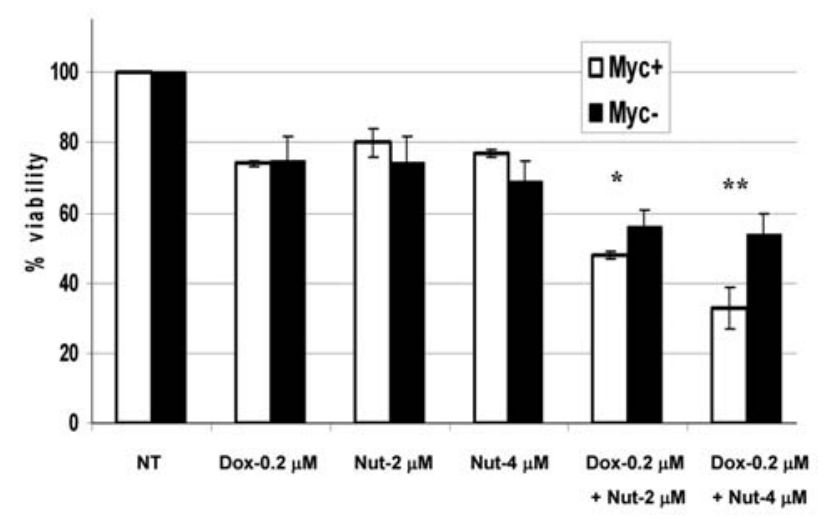

Figure 5. In a MycN-inducible cell line (SHEP-Tet21N), MycN interacts with combination treatments to strongly increase levels of pro-apoptotic proteins and enhance inhibition of proliferation. (A) $\mathrm{MycN}$ protein expression upregulates p53, p21 and Bax proteins in response to the nutlin-3/doxorubicin treatments over levels seen without $\mathrm{MycN}$ expression. (B) $\mathrm{MycN}$ expression in combination with nutlin-3/doxorubicin treatments increases the inhibition of cell proliferation, as measured by $24 \mathrm{~h}$ WST- 1 assay.

Proliferation was significantly reduced in the presence of MycN using $0.2 \mu \mathrm{M}$ doxorubicin $+2 \mu \mathrm{M}$ nutlin-3 $(\mathrm{P}<0.05)$ and $0.2 \mu \mathrm{M}$ doxorubicin $+4 \mu \mathrm{M}$ nutlin-3 $(\mathrm{P}<0.01)$.

\section{Discussion}

Previous studies have provided evidence that $\mathrm{MycN}$ expression in NB cells without $M Y C N$ amplification induces apoptosis (23). Others have shown that $\mathrm{MycN}$ expression and chemotherapeutics together function to block survival of NB (25). In this study, we have expanded these studies. We provide evidence that the combination treatment of nutlin- 3 and doxorubicin is able to upregulate expression of $\mathrm{MycN}$ protein, and that high levels of $\mathrm{MycN}$ in the absence of $M Y C N$ amplification interact with this combination to cooperatively induce apoptosis. Thus, this study addresses the upregulation of the pro-apoptotic $\mathrm{MycN}$ protein in response to a chemotherapeutic combination treatment, a finding not previously reported.

$\mathrm{MycN}$ is a $62-64 \mathrm{kDa}$ nuclear phosphoprotein, with a number of transcription initiation sites controlling basal levels of transcription, including those for the E2F transcription factors which regulate cell cycle progression $(8,28)$. It has long been known that the retinoblastoma $(\mathrm{Rb})$ tumor supppressor/ 
E2F pathway is an essential link between proliferation and apoptosis and Rogoff et al (29) found that E2F1 functions critically in apoptosis, acting, in part, to upregulate expression of Chk2, a checkpoint kinase. Chk2, in turn, is responsible for the phosphorylation and stabilization of E2F1 (30). In addition to the binding of the $\mathrm{MycN}$ promoter, E2F1 transactivates pro-apoptotic genes not only by direct interaction $(31,32)$, but also by regulating the splicing of caspases- 8 and -9 and $\mathrm{BH} 3$ family members (33). E2F1 has long been known to bind to the N-terminal region of $\mathrm{Mdm} 2$ and $\mathrm{Mdm} 2$ blocks the apoptotic activity of E2F1 by this binding (34). Kitagawa et al (34) demonstrated that E2F1 transcriptional activity is essential to nutlin-3-mediated apoptosis. Importantly, E2F1 expression is induced by a large number DNA damaging agents, including doxorubicin (35).

In the current study, we propose that the combined doxorubicin and nutlin-3 treatment induce DNA damage and block Mdm2 binding, leading to E2F1 stabilization and transactivation of pro-apoptotic genes, including $M Y C N$. Here, the binding of p53 and E2F1 to Mdm2 is inhibited by the single nutlin-3 treatment, while doxorubicin treatment alone induces checkpoint kinases by DNA damage, which, in turn, stabilizes free E2F1. The combination treatment promotes high level E2F1 binding to the MYCN promoter, enhancing MycN expression and this is borne out by our finding of increased expression of both E2F1 and MycN proteins following this treatment (Fig. 1B). We also demonstrate the upregulation of $\mathrm{MycN}$ at the mRNA level (Fig. 3A); this increase in transcription is also likely mediated by the combination treatment-induced high level expression of E2F1.

$\mathrm{MycN}$ overexpression in normal, non-oncogenic cells leads to death (36) and Myc is able to bind to the E-boxes of a number of genes for proteins in the apoptosis pathway including p53 and $\mathrm{BH} 3$-only proteins $(37,38)$. Increased expression of $\mathrm{MycN}$ often promotes caspase- 8 activation in neuroblastoma (40). Consistent with this, we noted higher levels of cleaved nucleosomes following the combination treatment (Fig. 1A), an effect that was partially reversed by the knock-down of $\mathrm{MycN}$ (Fig. 4B). It is also known that neuroblastomas frequently display mutation of genes directing apoptosis (27). Fulda et al have reported that the loss of caspase- 8 protein expression occurs in $75 \%$ of NB cases, although they find that this loss is not necessarily correlated with $M Y C N$ amplification (39).

In the current study of NB cell lines lacking $M Y C N$ amplification, we conjecture that initiator and effector caspase genes are neither mutated nor methylated and that the upregulation of $\mathrm{MycN}$ results in high level activation of intact apoptotic cascades. This apoptotic signaling leads to downstream expression of proteins normally seen during NB differentiation including CD44 and EFNB3 (40), by mechanisms that remain elusive; however, one plausible link is that factors that inhibit CD44 and EFNB3 expression in $M Y C N$-amplified NB are lacking in these non-MYCNamplified SH-SY5Y cells. The delay in upregulation of CD44 and EFNB3 genes following combination treatment (Fig. 3B) suggests roles as downstream effectors.

Thus, combined doxorubicin and nutlin-3 treatment may provide the basis for a novel therapy strategy for the majority of neuroblastomas lacking $M Y C N$ amplification, especially as greater insights into the molecular mechanisms involved are gained. We intend to further investigate the efficacy of combination nutlin-3 and doxorubicin for therapy of non$M Y C N$-amplified NB in our preclinical in vivo model of this malignancy.

\section{Acknowledgements}

We thank Anindya Dasgupta for helpful discussions. This study was supported by CURE Childhood Cancer, Inc.

\section{References}

1. Brodeur GM: Neuroblastoma: biological insights into a clinical enigma. Nat Rev Cancer 3: 203-216, 2003.

2. Combaret V, Gross N, Lasset D, Frappaz G, Peruisseau T, Philip D, Beck D and Favrot MC: Clinical relevance of CD44 cell-surface expression and $\mathrm{N}$-myc gene amplification in a multicentric analysis of 121 pediatric neuroblastomas. J Clin Oncol 14: 25-34, 1996

3. Tang XX, Robinson ME, Riceberg JS, Kim DY, Kung TB, Titus TB, Hayashi S, Flake AW, Carpentieri D and Ikegaki N: Favorable neuroblastoma genes and molecular therapeutics of neuroblastoma. Clin Cancer Res 10: 5837-5844, 2004.

4. Gross N, Balmas Bourloud K and Brognara CB: MYCN-related suppression of functional CD44 expression enhances tumorigenic properties of human neuroblastoma cells. Exp Cell Res 260: 396-403, 2000.

5. Tang X, Evans A, Zhao H, Cnaan A, Brodeur G and Ikegaki N: Association among EPHB2, TrkA and MYCN expression in low-stage neuroblastomas. Med Pediatr Oncol 36: 80-82, 2001.

6. Tang XX, Zhao H, Robinson ME, Cohen B, Cnaan A, London W, Cohn SL, Cheung N-KV, Brodeur GM, Evans AE and Ikegaki N: Implications of EPHB6, EFNB2, and EFNB3 expressions in human neuroblastoma. Proc Natl Acad Sci USA 97: 1093610941, 2000.

7. van Noesel MM and Versteeg R: Pediatric neuroblastomas: genetic and epigenetic 'Danse Macabre'. Gene 325: 1-15, 2004.

8. Thomas WD, Raif AA, Hansford L and Marshall G: N-myc transcription molecule and oncoprotein. Int J Biochem Cell Biol 36: 771-775, 2004.

9. Weiss W, Aldape K, Mohapatra G, Feuerstein B and Bishop JM: Targeted expression of MYCN causes neuroblastoma in transgenic mice. EMBO J 16: 2985-2995, 1997.

10. Lüscher B and Larsson L: The basic region/helix - loop - helix/ leucine zipper domain of Myc proto-oncoproteins: function and regulation. Oncogene 18: 2955-2966, 1999.

11. Manohar CF, Bray JA, Salwen HR, Madafiglio J, Cheng A, Flemming C, Marshall GM, Norris MD, Haber M and Cohn SL: MYCN-mediated regulation of the MRP1 promoter in human neuroblastoma. Oncogene 23: 753-762, 2004.

12. Coulter D, Blatt J, D'Ercole A and Moats-Staats BB: IGF-I receptor inhibition combined with rapamycin or temsirolimus inhibits neuroblastoma cell growth. Anticancer Res 28: 1509-1516, 2008.

13. Bell E, Premkumar RR, Carr J, Lu X, Lovat PE, Kees UR, Lunec J and Tweedle DA: The role of MYCN in the failure of MYCN amplified neuroblastoma cell lines to G1 arrest after DNA damage. Cell Cycle 5: 2639-2647, 2006.

14. Alaminos M, Mora J, Cheung N-KV, Smith A, Qin J, Chen L and Gerald WL: Genome-wide analysis of gene expression associated with MYCN in human neuroblastoma. Cancer Res 63: 4538-4546, 2003.

15. Opel D, Poremba T, Simon T, Debatin K-M and Fulda S: Activation of Akt predicts poor outcome in neuroblastoma. Cancer Res 67: 735-745, 2007.

16. Kang J, Rychahou PG, Ishola TA, Mourot JM, Evers BM and Chung DH: N-myc is a novel regulator of PI3K-mediated VEGF expression in neuroblastoma. Oncogene 27: 3999-4007, 2008.

17. Alvarado C, Abramowsky C, Gu L, Zhou M, Soe M, Sullivan K, George B, Schemankewitz E and Findley H: Expression of inhibitor-of-apoptosis protein (IAP) livin by neuroblastoma cells: correlation with prognostic factors and outcome. Pediatr Dev Pathol 8: 621-628, 2005. 
18. Slack A, Chen Z, Tonelli R, Pule M, Hunt L, Pession A and Shohet JM: The p53 regulatory gene MDM2 is a direct transcriptional target of MYCN in neuroblastoma. Proc Natl Acad Sci USA 102: 731-736, 2005

19. Kang J, Rychahou P, Ishola T, Qiao J, Evers B and Chung D: MYCN silencing induces differentiation and apoptosis in human neuroblastoma cells. Biochem Biophys Res Commun 351: 192-197, 2006

20. Nara K, Kusafuka T, Yoneda A, Oue T, Sangkhathat S and Fukuzawa M: Silencing of MYCN by RNA interference induces growth inhibition, apoptotic activity and cell differentiation in a neuroblastoma cell line with MYCN amplification. Int J Oncol 30: 1189-1196, 2007.

21. Tang XX, Zhao H, Kung B, Kim DY, Hicks SL, Cohn SL, Cheung N-K, Seeger RC, Evans AE and Ikegaki N: The MYCN enigma: significance of MYCN expression in neuroblastoma. Cancer Res 66: 2826-2833, 2006.

22. Cohn SL, London WB, Huang D, Katzenstein HM, Salwen HR, Reinhart T, Madafiglio J, Marshall GM, Norris MD and Haber M: MYCN expression is not prognostic of adverse outcome in advanced-stage neuroblastoma with nonamplified MYCN. J Clin Oncol 18: 3604-3613, 2000.

23. Fulda S, Lutz W, Schwab M and Debatin M: MycN sensitizes neuroblastoma cells for drug-induced apoptosis. Oncogene 18: 1479-1486, 1999.

24. van Noesel M, Pieters R, Voûte P and Versteeg R: The N-myc paradox: N-myc overexpression in neuroblastomas is associated with sensitivity as well as resistance to apoptosis. Cancer Lett 197: 165-172, 2003.

25. Albihn A, Mo H, Yang Y and Henriksson M: Camptothecininduced apoptosis is enhanced by Myc and involves PKCdelta signaling. Int J Cancer 121: 1821-1829, 2007.

26. Fulda S, Lutz W, Schwab W and Debatin K: MycN sensitizes neuroblastoma cells for drug-triggered apoptosis. Med Pediatr Oncol 35: 582-584, 2000

27. Strieder V and Lutz W: E2F proteins regulate MYCN expression in neuroblastomas. J Biol Chem 278: 2983-2989, 2003.

28. Kramps C, Strieder V, Sapetschnig A, Suske G and Lutz W: E2F and $\mathrm{Sp} 1 / \mathrm{Sp} 3$ synergize but are not sufficient to activate the MYCN gene in neuroblastomas. J Biol Chem 279: 5110-5117, 2004.

29. Rogoff HA, Pickering MT, Frame FM, Debatis ME, Sanchez Y, Jones S and Kowalik TF: Apoptosis associated with deregulated E2F activity is dependent on E2F1 and Atm/Nbs1/Chk2. Mol Cell Biol 24: 2968-2977, 2004.
30. Stevens C, Smith L and La Thangue NB: Chk2 activates E2F-1 in response to DNA damage. Nat Cell Biol 5: 401-409, 2003.

31. Monfared P, Winkeler A, Klein M, Li H, Klose A, Hoesel M, Waerzeggers Y, Korsching S and Jacobs AH: Noninvasive assessment of E2F-1-mediated transcriptional regulation in vivo. Cancer Res 68: 5932-5940, 2008.

32. Nahle Z, Polakoff J, Davuluri RV, McCurrach ME, Jacobson MD, Narita M, Zhang MQ, Lazebnik Y, Bar-Sagi D and Lowe SW: Direct coupling of the cell cycle and cell death machinery by E2F. Nat Cell Biol 4: 859-864, 2002.

33. Merdzhanova G, Edmond V, De Seranno S, Van den Broeck A Corcos L, Brambilla C, Brambilla E, Gazzeri S and Eymin B: E2F1 controls alternative splicing pattern of genes involved in apoptosis through upregulation of the splicing factor SC35. Cell Death Differ 15: 1815-1823, 2008.

34. Kitagawa M, Aonuma M, Lee SH, Fukutake S and McCormick F: E2F-1 transcriptional activity is a critical determinant of Mdm2 antagonist-induced apoptosis in human tumor cell lines. Oncogene 27: 5303-5314, 2008

35. Wang B, Liu K, Lin F-T and Lin W-C: A role for 14-3-3\{tau $\}$ in E2F1 stabilization and DNA damage-induced apoptosis. J Biol Chem 279: 54140-54152, 2004.

36. Nilsson JA and Cleveland JL: Myc pathways provoking cell suicide and cancer. Oncogene 22: 9007-9021, 2003.

37. Reisman D, Elkind NB, Roy B, Beamon J and Rotter V: c-Myc trans-activates the $\mathrm{p} 53$ promoter through a required downstream CACGTG motif. Cell Growth Differ 4: 57-65, 1993.

38. Cui H, Li T and Ding H-F: Linking of N-Myc to death receptor machinery in neuroblastoma cells. J Biol Chem 280: 9474-9481, 2005.

39. Fulda S, Poremba C, Berwanger B, Hacker S, Eilers M, Christiansen H, Hero B and Debatin K-M: Loss of caspase-8 expression does not correlate with MYCN amplification, aggressive disease, or prognosis in neuroblastoma. Cancer Res 66: 10016-10023, 2006.

40. Gross N, Beretta C, Peruisseau G, Jackson D, Simmons D and Beck D: CD44H expression by human neuroblastoma cells: relation to MYCN amplification and lineage differentiation. Cancer Res 54: 4238-4242, 2004. 